\title{
STOP TURNING A BLIND EYE: Tobacco smoking among Egyptian patients with schizophrenia
}

\author{
Lobna Azzam, Nahla Nagy, Ghada RA Taha, Mahmoud Elhabiby, \\ Hussien Elkholy, Mostafa Yosef

\section{Psychiatry Department, Faculty of Medicine, Ain Shams University, Egypt}

\section{INTRODUCTION:}

The relation between schizophrenia and smoking has been the subject of many studies for years. Some showed smoking to be associated with both positive and negative symptoms of schizophrenia, others found no relationship.

\section{SUBJECTS AND METHODS:}

This is a case control study. A randomly selected sample of 346 patients with schizophrenia, the cases group included the diagnosis of Schizophrenia and had been smokers for at least 12 months. Control group included 150 non schizophrenic subjects.

Assessment was done using the following. a) Structured Clinical Interview for DSM-IV Axis-I disorders (SCID) b) Positive and Negative Syndrome Scale (PANSS) c) Fagerström Test for Nicotine Dependence (FTND). d) Reasons for Quitting scale (RFQ)

\section{RESULTS:}

\begin{tabular}{|c|c|c|c|c|c|c|c|}
\hline \multicolumn{8}{|c|}{$\begin{array}{l}\text { Comparison between cases and controls regar } \\
\text { smoking and FTND }\end{array}$} \\
\hline & & \multicolumn{2}{|c|}{ Control } & \multicolumn{2}{|c|}{ Case } & \multicolumn{2}{|c|}{$t$ test } \\
\hline & & Mean & SD & Mean & SD & $P$ value & Sig. \\
\hline \multicolumn{2}{|c|}{ Age of smoking } & 23.93 & 5.89 & 22.15 & 6.17 & 0.003 & $\mathbf{S}$ \\
\hline \multirow{3}{*}{\multicolumn{2}{|c|}{ FTND }} & 7.12 & 1.43 & 6.60 & 1.56 & $<0.001$ & $s$ \\
\hline & & \multirow{2}{*}{$\mathbf{N}$} & \multirow{2}{*}{$\%$} & \multirow{2}{*}{$\mathbf{N}$} & \multirow{2}{*}{$\%$} & \multicolumn{2}{|c|}{ Chi Square } \\
\hline & & & & & & P value & Sig. \\
\hline \multirow[t]{4}{*}{ 1st Cig } & After $60 \mathrm{~min}$. & 17 & 11.3 & 64 & 18.5 & \multirow{4}{*}{$<000.1$} & \multirow{4}{*}{$\mathbf{s}$} \\
\hline & 30-60 min. & 52 & 34.7 & 52 & 15 & & \\
\hline & 6-30 min. & 59 & 39.3 & 95 & 27.5 & & \\
\hline & Within $5 \mathrm{~min}$. & 22 & 14.7 & 135 & 39 & & \\
\hline \multirow{4}{*}{$\begin{array}{l}\text { No. of } \\
\text { cig/d }\end{array}$} & $<10$ & 3 & 2 & 78 & 22.5 & \multirow{4}{*}{$<0.001$} & \multirow{4}{*}{$\mathbf{s}$} \\
\hline & $11-20$ & 116 & 77.3 & 130 & 37.6 & & \\
\hline & $21-30$ & 17 & 11.3 & 95 & 27.5 & & \\
\hline & $>30$ & 14 & 9.3 & 43 & 12.4 & & \\
\hline
\end{tabular}

\begin{tabular}{|c|c|c|c|c|}
\hline \multicolumn{5}{|c|}{$\begin{array}{l}\text { Correlation between symptoms of schizophrenia (PANSS) AND the number of } \\
\text { cigarettes and time spent before first cigarette }\end{array}$} \\
\hline & \multicolumn{2}{|c|}{$\begin{array}{l}\text { Number of cigarettes } \\
\qquad N=346\end{array}$} & \multicolumn{2}{|c|}{$\begin{array}{l}\text { Time before 1st cigarette } \\
\qquad N=346\end{array}$} \\
\hline & rs & p & rs & p \\
\hline Delusions & 0.147 & 0.006 & -0.160 & 0.003 \\
\hline Conceptual disorganization & 0.428 & $<0.001$ & -0.417 & $<0.001$ \\
\hline Hallucinations & 0.213 & $<0.001$ & -0.201 & $<0.001$ \\
\hline Excitement & 0.287 & $<0.001$ & -0.286 & $<0.001$ \\
\hline Grandiosity & 0.055 & 0.307 & -0.085 & 0.115 \\
\hline Suspiciousness/Persecution & 0.085 & 0.115 & -0.106 & 0.048 \\
\hline Hostility & 0.185 & 0.001 & -0.227 & $<0.001$ \\
\hline Blunt affect & 0.018 & 0.740 & -0.002 & 0.968 \\
\hline Emotional withdrawal & -0.010 & 0.852 & 0.042 & 0.434 \\
\hline Poor rapport & -0.029 & 0.591 & 0.049 & 0.361 \\
\hline $\begin{array}{l}\text { Passive/apathetic social } \\
\text { withdrawal }\end{array}$ & 0.003 & 0.950 & 0.005 & 0.922 \\
\hline Difficulty in abstract thinking & 0.035 & 0.513 & 0.011 & 0.837 \\
\hline $\begin{array}{l}\text { Lack of spontaneity \& flow of } \\
\text { conversation }\end{array}$ & -0.019 & 0.726 & 0.025 & 0.643 \\
\hline Stereotyped thinking & 0.042 & 0.434 & -0.030 & 0.584 \\
\hline Somatic concern & 0.079 & 0.143 & -0.125 & 0.020 \\
\hline Anxiety & 0.157 & 0.003 & -0.138 & 0.010 \\
\hline Guilt feelings & 0.013 & 0.816 & -0.055 & 0.306 \\
\hline Tension & -0.051 & 0.343 & 0.053 & 0.326 \\
\hline Mannerism \& posturing & 0.099 & 0.067 & -0.087 & 0.107 \\
\hline Depression & 0.035 & 0.519 & -0.020 & 0.704 \\
\hline Motor retardation & -0.178 & 0.001 & 0.165 & 0.002 \\
\hline Uncooperativeness & 0.011 & 0.843 & 0.001 & 0.985 \\
\hline Unusual thought content & -0.035 & 0.518 & 0.031 & 0.563 \\
\hline Disorientation & 0.045 & 0.408 & -0.087 & 0.106 \\
\hline Poor attention & -0.138 & 0.010 & 0.157 & 0.003 \\
\hline Lack of judgment \& insight & 0.029 & 0.587 & -0.008 & 0.883 \\
\hline Disturbance of volition & 0.096 & 0.074 & -0.076 & 0.157 \\
\hline Poor impulse control & 0.226 & $<0.001$ & -0.219 & $<0.001$ \\
\hline Preoccupation & 0.150 & 0.005 & -0.139 & 0.010 \\
\hline Active social avoidance & 0.014 & 0.796 & -0.043 & 0.422 \\
\hline
\end{tabular}

\section{CONCLUSION:}

Tobacco is one of the major contributors to major health issues in patients with schizophrenia. It seems that there is some sort of a link between some positive symptoms of schizophrenia and severity of tobacco dependence, number of cigarettes and morning smoking preference. 